\title{
Surgical treatment of atrial myxomas, 6 years' experience via standard sternotomy
}

\author{
Hamdy D. Elayouty ${ }^{1}$, Mursi M. Amin ${ }^{2}$, Ahmed M. Sami ${ }^{3}$, Mohamed A. Alboghdadi ${ }^{4}$ \\ 1. Depart of Cardiothoracic Surgery, Suez Canal University/Egypt and Hadhramout University, Almukalla, Yemen. 2. \\ Depart of Cardiothoracic Surgery, Suez Canal University, Ismailia, Egypt. 3. Depart of Cardiothoracic Surgery, Ain Shams \\ University, Cairo, Egypt. 4. Depart of Cardiology, Hadhramout University, Almukalla and Alaml Medical Center, Taez, \\ Yemen
}

Correspondence: Hamdy D. Alayouty. Address: Department of Chest Surgery -Suez Canal University Hospitals, Ismailia, Egypt. Telephone: 002-013-727-1234. Fax: 009-670-536-3022. E-mail: h.dosoky@yahoo.com

Received: April 29, 2012

DOI : $10.5430 /$ jst.v2n4p32
Accepted: J une 17, 2012

URL: http://dx.doi.org/10.5430/jst.v2n4p32

\section{Abstract}

Introduction: Myxomas are the most significant cardiac tumors, not only because of their frequency but also because the potential for total cure after surgical removal is high. The major determinant of this cure is supposed to be complete excision of these atrial myxomas whatever the pathological or clinical features of the tumor mass.

Objectives: The aim was to evaluate the result of complete surgical excision of myxomas having divergent clinical presentations and different pathological features.

Methods: Current prospective study included fourteen female and eight male patients with mean age of $42 \pm 2.3$ years. Left atrial myxomas were found in 18 (82\%) and right atrial ones in four (18\%) cases. Frequent clinical findings included fatigue: in 16 (73\%), dyspnea: in 14 (84\%) and prolonged fever: in four cases (18\%). Diastolic murmur at the apex was detected in eight patients but at the tricuspid area in two cases. Pansystolic murmur in two at the apex. Electrocardiography showed atrial fibrillation in three and ventricular hypertrophy in two cases. Two-dimensional trans-thoracic echocardiography diagnosed myxoma in 20 cases; the remaining two needed trans-esophageal echocardiography. Two had mitral regurgitation. Preoperative coronary angiography detected significant coronary artery disease that indicated coronary angioplasty in two cases. Twenty two patients had open heart surgery via median sternotomy for excision of the myxomas. Two of them had mitral valve repair in the same session.

Results: Cardiopulmonary bypass time ranged between 75 and 115 minutes. Aortic cross clamp time ranged between 45 and 60 minutes. Operative findings: the tumors arose from the atrial septum in 19 cases, from left atrial wall in one and from the mitral valve annulus in two cases. Dimensions of the masses ranged between 3 and 7 centimeters. Myxomas were pedunculated in 18 patients (82\%) and sessile in four (18\%) tumors. Pathologically, all masses were benign true neoplasms. There were no early or late hospital deaths or strokes, There were two cases of wound infection. The mean follow up was $5.6 \pm 0.5$ years. Patients did not show any recurrences, new arrhythmias or septal leakage.

Conclusions: Long term follow up after complete excision of atrial myxomas with a safety margin of surrounding atrial tissue showed no recurrences, new arrhythmias or septal leakage in spite of preoperative divergent clinical and pathological features. 


\section{Key words}

Atrial myxoma, Echocardiography, Extirpation of myxomas via standard sternotomy

\section{Background}

These many-faceted tumors account for about half of all primary cardiac neoplasms ${ }^{[1]}$. This cardiac neoplasm is "The Great Imitator”. The tumor can assume the guise of valvular or pericardial disease, congestive heart failure or pulmonary hypertension, embolization or arrhythmia, hemolytic anemia or infectious endocarditic ${ }^{[1]}$.The patient`s presenting symptom may be stroke, syncope, chest pain, fever, or loss of weight. Cardiac tumors enter into the differential diagnosis of virtually every cardiovascular syndrome. Cardiologists should think of them in the early stages of physical assessment of the patient because the most common cardiac tumor, atrial myxoma, is "devastating but curable" ${ }^{[2]}$. Yet, it is gloomily claimed that only 5 to 10 percent of cardiac tumors are clinically diagnosable ${ }^{[3]}$. Also, recently with minimally invasive surgical techniques, there are recurrent cases. Therefore, we are going to explore our experience with this Great Imitator to evaluate the results of complete surgical excision of myxomas having divergent clinical presentations and different pathological features ( shape, site, size, number of masses, etc. ) using the median sternotomy incision.

\section{Patients and methods}

Current prospective descriptive study included 22 patients with myxomas of the heart. From 1st January 2005 to 31st Dec. 2011, 14 females and 8 males, with mean age of $42 \pm 2.3$ years, were diagnosed to have myxomas of the heart. Trans-thoracic echocardiography confirmed the diagnosis in 20 cases. Trans-esophageal echocardiography was necessary to confirm the diagnosis in two cases. Coronary artery catheterization was indicated preoperatively, for patients beyond the age of forty years (13 cases). Two had coronary angioplasty. Open heart surgery via median sternotomy was the procedure of choice to achieve adequate excision of the tumors.

The operation consisted of excision of the tumor with a generous portion of atrial septum or wall. The operative approach included bicaval cannulation and snaring. Incision into the appropriate atrium allowed trans-septal extirpation of the mass with a part of atrial septum. The surgeon made the incision carefully avoiding injury to the Koch`s triangle. Patch reconstruction of the atrial septum was required in all patients. The atrial defect was closed with a native pericardial (in eight cases) or a Dacron patch (in 14 cases). Mitral valve replacement was done in two cases. Biatrial approach was done in four cases with large tumors. An incision is made in the left atrial wall posterior to the interatrial groove and the tumor and its attachments were identified without any mobilization of the tumor. An oblique right atriotomy was made, the right chambers were explored, and then the superior rim of the left atriotomy was retracted to allow complete and safe excision of the mass. With the biatrial approach, both atria and ventricles were carefully inspected for tumor fragments or other myxomas. Biatrial approach was also done for patients in need for mitral valve repair.

\section{Results}

Left atrial myxomas were found in 18 patients and right atrial ones in four cases. The most frequent clinical features were fatigue in 16 (73\%), shortness of breath in 14(63\%) and prolonged fever in four (18\%). On auscultation, diastolic murmur over the mitral area was detected in eight and in two over the tricuspid area. Electrocardiograph showed atrial fibrillation in three and cardiomegaly in two cases. Two-dimensional trans-thoracic echocardiography diagnosed myxoma in 20 cases. The remaining two needed trans-esophageal echocardiography, Dimensions of the masses were about $3 \times 5 \mathrm{~cm}$ in $82 \%$ and $5 \times 7 \mathrm{~cm}$ in $18 \%$.

Two had mitral regurgitation. Various degrees of pulmonary hypertension were found in all left atrial myxomas. No one case was accidentally discovered or diagnosed only on table (in the theater).Twenty patients had open heart surgery for 
excision of the myxoma. Two had mitral valve repair (annulopalsy and repair of anterior leaflet) along with extirpation of the myxomas. Operative findings established that the tumors arose from the atrial septum in 19 cases. The tumor arose from left atrial wall in one and from the mitral valve annulus in two cases. Eighteen masses were pedunculated and 4 were sessile. The four right atrial myxomas were more solid and each one involved a greater amount of atrial septum $(n=3)$ or atrial wall $(n=1)$ than did the left atrial ones. Pathological reports described benign true neoplasms in all cases. Each mass was composed of a basophilic myxoid matrix containing polygonal cells which were frequently stellate multinuclear cells. Other cells were found such as plasma cells, lymphocytes, and mast cells. Foci of calcification were seen in three cases (15\%).

Mean stay in intensive care unit was $1 \pm 0.5$ days and hospital stay was $10 \pm 2.6$ days. Two patients had wound infections that were controlled before discharge. The mean follow up was $5.6 \pm 0.5$ years and ranged between 60 and 72 months. All patients were symptomless, New York Heart Assoceation-class1 and without any clinical or echocardiographic signs of recurrence, new arrhythmias or septal leakage (see Table 1).

Table 1. Perioperative complications

\begin{tabular}{lll}
\hline Results of surgery & Number of patients & Percentage (\%) \\
\hline Recurrence & 0 & ---- \\
Wound infection & 2 & 9 \\
Mortality & 0 & ---- \\
\hline
\end{tabular}

\section{Discussion}

Atrial myxomas account for $35 \%$ to $50 \%$ of primary cardiac tumors ${ }^{[4]}$. In this series females represented $63 \%$ of the sample size and most of patients were in the 3rd and 4th decades of life and this agrees with sex differences and age distribution reported in literature.

Most experts consider atrial myxomas true neoplasms on the basis of histological, ultra structural, electron microscopic and tissue culture studies. There is a report of $3 \times 2 \mathrm{~cm}$ right atrial myxoma developing at the site of trans-septal catheterization for balloon mitral valvuloplasty. Such masses were described as reactive myxomas and not true neoplasms ${ }^{[5]}$.

Table 2. Clinical and pathological features of atrial myxomas in current series

\begin{tabular}{lll}
\hline Manifestation & Number of patients & Percentage (\%) \\
\hline Male patients & 8 & 36 \\
Female patients & 14 & 63 \\
Left atrial masses & 18 & 82 \\
Right atrial masses & 4 & 18 \\
Pyrexia & 4 & 18 \\
Dyspnea & 14 & 63 \\
Fatigue & 16 & 73 \\
Atrial fibrillation & 3 & 13 \\
Cardiomegaly & 2 & 9 \\
Pedunculated mass & 18 & 82 \\
Size of tumors 3 x 5 cm & 18 & 82 \\
Size of masses 5 x 7 cm & 4 & 18 \\
Pedunculated masses & 18 & 82 \\
Sessile masses & 4 & 18 \\
\hline
\end{tabular}


Two-dimensional echocardiography is the diagnostic procedure of choice. Most of them are benign and can be resected. Seventy eight percent occur in the left atrium with or without involvement of the mitral valve or mechanically affecting the valve. The remaining are mostly in the right atrium (18\%) and few (4\%) in the ventricles. This is similar to our clinical findings.

Seven percent are inherited (familial myxomas), that may grow in any of the four cardiac chambers ${ }^{[4,5]}$. The diameters of masses in current study ranged between 3 and $7 \mathrm{~cm}$. Reported dimensions in literature ranged between 0.5 and $13 \mathrm{~cm}^{[5]}$. Intra atrial mass can be an organized thrombus after closure of a congenital atrial septal defect ${ }^{[6]}$ or developing at the site of trans-septal catheterization. That is why careful history taking is mandatory.

This great imitator tumor assumes importance because it is dramatically curable. Furthermore, if a myxoma is present but the diagnosis is not made, the patient remains exposed to the risks of embolization and sudden death ${ }^{[7]}$. In view of the dangers of embolization and sudden death, surgical resection should be performed promptly. Clinical manifestations that helped us to pick up our patients are shown in Table 2.

Also, obstruction of a valve orifice by a pedunculated myxoma or hemorrhage into a myxoma indicated emergency operation and reported by Uday Sonker and his colleagues, $2009^{[8]}$.

Another complication was described by George Anagnostopoulos and others, 2004. They reported that right atrial myxoma was a rare but potentially curable cause of Budd-Chiari syndrome ${ }^{[4]}$.

The clinical diagnosis and most of the complications can be confirmed or excluded with echocardiography. Echocardiography has replaced cardiac catheterization as the mainstay of diagnosis because of its noninvasive advantages ${ }^{[9]}$. Although myxomas are rare, they should be considered in the differential diagnosis of patients with suspected mitral valve disease, congestive heart failure or constitutional disturbances and embolization among patents in sinus rhythm ${ }^{[10]}$.

The diagnosis of right atrial tumors can be difficult and their resection presents difficulties for the placement of the venous cannulae. In such cases, we used a single cannula in the superior vena cava until fibrillation and then inserted a cannula into the inferior vena cava as described by Soma and John, $2000{ }^{[9]}$. .In five cases, we used the biatrial approach. The advantages of biatrial approach are the identification of the site of [attachment, inspection of the four cardiac chambers, the adequate irrigation, and the prevention of fragmentation and intra-operative embolization. The final objective of the surgeon is to lessen the possibility of recurrence and the incidence of postoperative arrhythmias. The biatrial approach adds further safety and completeness of removal of the tumor in order to lessen the possibility of future recurrence, and by the precise limited excision of the attachment possibly lessens the incidence of postoperative arrhythmias ${ }^{[11]}$.

Robotic Surgery offers innovative approach to treat atrial myxomas and thrombi through the use of the da Vinci Surgical System. For the clinically appropriate patient, the da Vinci minimally-invasive procedure offers a number of potential benefits, including: less post-operative pain, less scarring less risk of infection, less anesthesia, less blood loss, shorter hospital stay, faster and more complete recovery, and quicker return to normal daily activities ${ }^{[12]}$. But, should young surgeons or surgeons in developing countries who had started on-pump surgery within the last two or three decades, start treatment of atrial myxomas with minimally invasive techniques? We believe that it is not necessary. We are presenting work with no recurrence after 6 years of regular follow up, no strokes or septal leakage. The latter two complications were reported after minimally invasive and robotic technique. The reported recurrence rate for benign myxomas was 7 to $9 \%$. The interval from first operation to recurrence has ranged from 7 months to 5 years ${ }^{[13]}$. Atrial myxomas were successfully treated with minimally invasive surgery in many centers, but with recurrent cases, strokes and septal leakage. Kotylo et.al, 1991 reported that myxomas with aberrant DNA content (aneuploid tumors) determined by flow cytometry were associated with aggressive biologic behavior and are probably more likely to recur ${ }^{[14]}$. 
The mass was excised with a full thickness of intra-atrial septal attachment with a safety margin and frozen sections from margins of some tumor masses were examined to prove margins free of tumor matrix or cells. Then, induced interatrial septal defect was closed with a native pericardial or a Dacron patch.

Postoperative wound infection evolved in two cases: one with superficial wound infection that was rapidly controlled with local antibiotic cream. The second patient had superficial infection that spread to deeper tissues in a patient with type 1 diabetes melletus, the blood sugar was promptly controlled and antibiotics administered for 7 days according to culture and sensitivity. Wound infection was properly controlled before discharge.

Echography was done yearly during the follow up. The mean follow up in our series was $5.6 \pm 0.5$ years. Cleveland and his

colleagues reported that recurrence is rare and usually occurs within 48-month period ${ }^{[15]}$. Regular follow up showed no recurrences, new arrhythmias or septal leakage among our patients.

\section{Conclusion}

Excision of the tumor with a safety margin of surrounding atrial tissue and long term follow up showed no recurrences, new arrhythmias or septal leakage among patients with divergent clinical and pathological features of their myxomas.

\section{Acknowledgements}

Thanks to Prof. Fathy Makledy (MRCP) - Professor of Cardiology and previous Vice President of Suez Canal University, for his great help in diagnosis and follow up of the patients.

\section{References}

[1] Wold L.E. and Lie J.T. Cardiac myxomas: a clinicopathological profile. Am. J. Pathol. 1980; 101: 219. PMid:7446701

[2] Peters M, Hall R, Cooley D, Leachman R and gorcia E. The clinical syndrome of atrial myxoma JAMA. 1974; 230: 695-701. http://dx.doi.org/10.1001/jama.1974.03240050023018

[3] Bloor C. M. and O`Rourke R, A. Cardiac tumors: clinical presentations and pathologic correlation Curr.Probl,Cardiol. 1984 ; 9: 7. PMid:6386348

[4] George K. Anagnostopoulos, George Margantinis, Panagiotis Kostopoulos, Glyceria Papadopoulou, Athanassios Roulias, George Sakorafas, Nikolaos Liassis Budd-Chiari syndrome and portal vein thrombosis due to right atrial myxoma Ann Thorac Surg. 2004; 78: 333-334. PMid:15223461

[5] Haught W.N., Alexander J.A.and Conti C.R. Familial recurring cardiomyxoma. Clin. Cardiol. 1991; $14: 692$. http://dx.doi.org/10.1002/clc.4960140813

[6] Ahmad Y. Sheikh, Sonja Schrepfer, William Stein, Joan West, Jane Lombard, Tom Burdon, Bob Pinsker, Marc P. Pelletier Right Atrial Mass After Primary Repair of an Atrial Septal Defect: Thrombus Masquerading as a Myxoma Ann Thorac Surg. 2007; 84: 1742-1744.

[7] Sutton M. G. St. J., Mercier L. A., Giuliana E.R. and Lie J.T. Atrial myxoma: a review of clinical experience in 40 patients Mayo Clin.Proc. 1980; 55: 371. PMid:7382545

[8] Uday Sonker, Geoffrey T.L. Kloppenburg, Esther A. Knoop, Cornelis A. Seldenrijk, Wim J. Morshuis Emergency Surgery for Acute Mitral Valve Obstruction Resulting From Hemorrhage Within a Left Atrial Myxoma Ann Thorac Surg. 2009; 87: 636-638.

[9] Soma Guhathakurta and John P. Riordan. Surgical Treatment of Right Atrial Myxoma. Texas Heart Inst J. 2000; 27: 61-3. PMid:10830633

[10] St John Sutton M, Mercier L, Giuliani E, Lie J. Atrial myxomas: a review of clinical experience in 40 patients Mayo Clin Proc. 1980; 55: 371-9. PMid:7382545

[11] Kyriakos St. Rammos Modified Biatrial Approach for the Surgical Excision of Left Atrial Myxomas Ann Thorac Surg. 1997; 64: 274-275.

[12] Alexander L, Rachel E, Mark J, Jonathan Y, Faisal H, Craig R. Long-term outcomes with a minimally invasive approach for resection of cardiac masses. Am Thorac Surg. 2010; 90: 1251-55. PMid:20868822

http://dx.doi.org/10.1016/j.athoracsur.2010.05.050 
[13] Changging G, Ming Y, Gang W, Jiail W, Cangsong X. Yang W. Excision of atrial myxoma using robotic technology J Thoracic CardivasularSurg. 2010; 139: 1282-1285.

[14] Kotylo P, Kennedy J, Waller B and Sample R DNA analysis of atrial myxomas. Chest. 1991; 99: 1203-6. PMid:2019179 http://dx.doi.org/10.1378/chest.99.5.1203

[15] Cleveland D C, Westaby S, Karp R B. Treatment of intra-atrial cardiac tumors. JAMA. 1983; 249: 2799-2802. http://dx.doi.org/10.1001/jama.1983.03330440037028 